\title{
Analytische und technische Zuverlässigkeit des ELT-8 Laser Hämatologie Zellzählers
}

\author{
Von W. Bauersfeld, E. Knoll und H. Wisser \\ Abteilung für Klinische Chemie/Labormedizin des Robert-Bosch-Krankenhauses Stuttgart
}

(Eingegangen am 19. Juni/26. Oktober 1984)

Zusammenfassung: Der ELT-8 Laser Hämatologie-Zellzähler ist ein vollmechanisiertes Gerät, das die Zellzählung mit Hilfe der an Partikeln hervorgerufenen Vorwärtsstreuung eines Helium-Laserstrahles durchführt.

Die Praktikabilität des Gerätes wird als sehr gut beurteilt. Das Probenvolumen ist mit $100 \mu \mathrm{l}$ sehr niedrig. Die Einarbeitungszeit ist wegen der Einfachheit der Bedienung kurz. Die Ausfallzeiten im zweiten Betriebsjahr des Gerätes betrugen durchschnittlich 8 Stunden/Monat.

Die Präzisionen der Messungen liegen je nach Kenngröße zwischen 0,5 und 4\% in der Serie und 1-9\% von Tag zu Tag. Die höchste Streuung wurde für die Thrombocyten ermittelt. Dies könnte allerdings durch Instabilitäten der Kontrollprobe bedingt sein. Eine Verschleppung konnte bei Messung von Proben unterschiedlicher Konzentration der zu bestimmenden Kenngrößen ausgeschlossen werden. Lipämie und Hämolyse der Proben können zu Fehlbestimmungen bzw. zu Fehlinterpretationen führen. Als Einflußgrößen können Kälteagglutine gegen Erythrocyten und Thrombocyten sich störend bemerkbar machen. Sie sind erkennbar an diskrepanten Ergebnissen von Erythrocytenzahl und Hämoglobin sowie MCV bzw. „Thrombopenien“ bei fehlender klinischer Symptomatik (Pseudothrombopenie). In solchen Fällen kann ein Histogramm hilfreich sein. Es wurden Untersuchungen zur Stabilität der Blutproben bei einer Aufbewahrung von 72 verschiedenen Blutproben über 4 Tage bei $4^{\circ} \mathrm{C}$ durchgeführt. Die Kenngrößen des roten Blutbildes zeigten eine hohe Stabilität, während die Thrombocyten und Leukocyten zum Teil unregelmäßige Veränderungen aufwiesen. Das Gerät arbeitet linear bis zu folgenden Grenzwerten: Leukocyten $85 \times 10^{9} / 1$, Erythrocyten $8 \times 10^{12} / 1$, Thrombocyten $10^{12} / 1$ und Hämoglobin $230 \mathrm{~g} / \mathrm{l}$.

Die Übereinstimmung der Messungen mit dem ELT-8 und konventionellen Verfahren ist sehr gut, wie folgende Regressionsgeraden für jeweils 40 verschiedene Proben ausweisen: Leukocyten $\left(10^{9} / 1\right): y=1,116 x$ $-0,532, r=0,985$; Erythrocyten (1012/1): $y=0,992 x+0,194, r=0,978 ;$ Hämoglobin $(\mathrm{g} / \mathrm{l}): \mathrm{y}=0,967 \mathrm{x}+$ $2,758, r=0,987$ und Thrombocyten $\left(10^{9} / 1\right): y=1,015 x+9,19, r=0,945$.

\section{Analytical and technical reliability of the ELT-8 laser haematological counter}

Summary: The ELT-8 laser haematological cell counter is a fully mechanized instrument, which measures the forward scatter of light from a helium-neon laser caused by suspended particles.

The apparatus has high practicability, and uses a very small sample volume of $100 \mu$. The operational procedure is simple and quickly learned. In the second year of operation, down time for the instrument was, on average, 8 hours/month.

Depending on the parameter, the precision of the measurements are between 0.5 and $4 \%$ in series, and $1-9 \%$ day-to-day. The highest scatter of results was shown for thrombocyte counting, but this may be due to instability of the control samples. There was no carry over between samples containing different concentrations of any of the measured parameters. Lipaemia and haemolysis of samples can lead to false results 
or false interpretation of results. Cold agglutinins, active against erythrocytes and thrombocytes, can cause significant interference, and this influence can be recognized from discrepancies between the erythrocyte count and haemoglobin concentration or mean corpuscular volume (MCV), and from the presence of thrombopenia in the absence of clinical symptoms (pseudothrombopenia). In such cases a histogram is useful. The stability of 72 blood samples was investigated during storage for 4 days at $4^{\circ} \mathrm{C}$. The erythrocyte count was very stable, whereas thrombocyte and leukocyte counts sometimes showed irregular variations.

The apparatus gives a linear response up to the following limiting values: leukocytes $85 \times 10^{9} / 1$, erythrocytes $8 \times 10^{12} / 1$, thrombocytes $10^{12} / \mathrm{l}$, haemoglobin $230 \mathrm{~g} / \mathrm{l}$.

Results from the ELT-8 show very good agreement with those from conventional methods, as shown by the following regression lines determined from the analysis of 40 different samples: leukocytes $\left(10^{9} / 1\right): y=1.116 x$ $-0.532, r=0.985$; erythrocytes $\left(10^{12} / 1\right): y=0.992 x+0.194, r=0,978 ;$ haemoglobin $(g / l): y=0.967 x+$ $2.758, \mathrm{r}=0.987$; thrombocytes $\left(10^{9} / \mathrm{l}\right): \mathrm{y}=1.015 \mathrm{x}+9.19, \mathrm{r}=0.945$.

\section{Einführung}

Zur Bestimmung der Kenngrößen des kleinen Blutbildes steht eine Reihe von Geräten zur Verfügung. Bezüglich des Meßprinzips kann man zwei verschiedene Gerätetypen unterscheiden: Solche, die Leitfähigkeitsveränderungen - bedingt durch die Partikel in der Suspension - und solche, die die Streustrahlung eines Lasers an den Partikeln messen. Diese Techniken erlauben die Messung einer großen Anzahl von Proben mit, im Vergleich zu den konventionellen Verfahren, hoher Präzision.

In den folgenden Ausführungen soll über unsere Erfahrungen mit dem ELT-8 Laser HämatologieZellzähler berichtet werden. Für die Beurteilung des Gerätes wurden analytische Kriterien (Präzision, Richtigkeit, Linearität), technische Kriterien (Ausfallzeiten, Reparaturkosten, Reagenzienkosten) und Praktikabilitätskriterien (Handhabung des Gerätes, Einarbeitungszeit, Sicherheit) herangezogen. Des weiteren sollte geklärt werden, inwieweit die Bestimmung der Größenverteilungskurven von Erythrocyten, Leukocyten und Thrombocyten bei verschiedenen Erkrankungen von diagnostischem Interesse ist.

\section{Methodik}

Der ELT-8 Hämatologie-Zellzähler') ist ein automatisches Zellzählgerät, das die Zellzählung mit Hilfe der an Partikeln hervorgerufenen Vorwärtsstreuung eines Helium-Neon-Laserstrahlers durchführt. Das Streulicht wird in elektrische Impulse umgewandelt. Die Zählung der verschiedenen Zellen (Erythrocyten, Leukocyten, Thrombocyten) erfolgt nach Anzahl und Größe der verschiedenen elektrischen Impulse. Das Hämoglobin wird photometrisch mit der Cyanhämiglobinmethode bestimmt. Der Hämatokrit wird aus dem Integral der Impulse der Erythrocyten erhalten. Die Unterscheidung von Erythrocyten, Leukocyten und Thrombocyten geschieht, indem Leukocyten nach Hämolyse der Erythrocyten in einem Kanal und Erythrocyten sowie Thrombocyten in einem zweiten Kanal gemessen

$\left.{ }^{1}\right)$ Vertrieb in Deutschland: Dr. Molter GmbH, Heidelberg. werden. Die Unterscheidung von Thrombocyten und Erythrocyten erfolgt aufgrund von Größe und Breite des Streulichtsignals. Das Probenvolumen beträgt $100 \mu \mathrm{l}$. Dies ist für Routineuntersuchungen sehr günstig, da bei Verstopfungen des Gerätes oder auch Fehlmessungen Wiederholungsuntersuchungen ohne Schwierigkeiten durchgeführt werden können. Der Probendurchsatz des Gerätes mit etwa 50 Proben/Stunde ist nicht sehr hoch. Andererseits sind bei einer zu hohen Probenfrequenz notwendige Plausibilitätskontrollen durch den Bediener des Gerätes nicht mehr möglich.

Bei den Vergleichsmessungen erfolgte die Zählung der Erythrocyten und Leukocyten mittels geeichter Neubauer-Kammern nach Verdünnung mit Hayem's Reagenz bzw. Türk'scher Lösung im Lichtmikroskop. Die Thrombocyten wurden mit Pla$x^{10}{ }^{10}$ im Phasenkontrastmikroskop ausgezählt. Die Hämoglobinbestimmung wurde mittels der Cyanhämiglobinmethode manuell durchgeführt. Der Hämatokrit wurde mit einer Hämatokritzentrifuge ermittelt.

Die Kalibrierung des ELT-8 erfolgte mit Normocell ${ }^{10}$ (Fa. Molter, Heidelberg).

\section{Ergebnisse und Diskussion}

\section{Präzision}

Die Präzision der Messungen mit dem ELT-8 wurde durch Mehrfachmessung von Kontrollproben ${ }^{2}$ ) niedriger, normaler und erhöhter Konzentration der zu bestimmenden Kenngrößen ermittelt. Die Ergebnisse dieser Messungen für jeweils $n=20$ sind in Tabelle 1 zusammengefaßt.

Die Streuungen liegen für die Messungen in der Serie zwischen 0,5 und $4,0 \%$ und für die Messuingen von Tag zu Tag zwischen 1,0 und 9,0\%. Die größten Streuungen wurden für die Thrombocytenzählung ermittelt, wobei allerdings eine unverhältnis̀mäßig starke Zunahme für die Streuung von Tag zu Tag im Vergleich zu der Streuung in dèr Sèrie festgestellt wurde. Die ist möglicherweise durch Instabilitäten

${ }^{2}$ ) Control Blood, AHS, München und Thrombocyten-Standard, KABE-Labortechnik GmbH; Nümbrecht-Elsenroth. 
Tab. 1. Ergebnisse der Bestimmung der Präzision in der Serie und von Tag zu Tag durch Zwanzigfachmessung von Kontrollproben niedriger, normaler und hoher Konzentration der zu bestimmenden Parameter (MCV = mittleres korpuskuläres Volumen, $\mathrm{MCH}=$ mittleres korpuskuläres Hämoglobin. $\mathrm{MCHC}=$ mittlere korpuskulärc Hämoglobinkonzentration).

\begin{tabular}{|c|c|c|c|c|c|c|}
\hline \multirow[t]{2}{*}{ Parameter } & \multicolumn{3}{|c|}{$\begin{array}{l}\text { Präzision in der Serie } \\
(n=20)\end{array}$} & \multicolumn{3}{|c|}{$\begin{array}{l}\text { Präzision von Tag zu Tag } \\
(n=20)\end{array}$} \\
\hline & $\overline{\mathbf{x}}$ & s & $v(\%)$ & $\overline{\mathbf{x}}$ & $\mathbf{s}$ & $v(\%)$ \\
\hline \multicolumn{7}{|l|}{ Niedrige Konzentration } \\
\hline $\begin{array}{l}\text { Lcukocyten }\left(10^{9} / \mathrm{l}\right) \\
\text { Erythrocyten }\left(10^{12} / 1\right) \\
\text { Hämoglobin }(\mathrm{g} / \mathrm{l}) \\
\text { Hämatokrit } \\
\text { MCV (f) } \\
\text { MCH (pg) } \\
\text { MCHC (g/l) } \\
\text { Thrombocyten }\left(10^{9} / 1\right)\end{array}$ & $\begin{array}{c}2.49 \\
2.55 \\
76.55 \\
0.208 \\
81.6 \\
30.01 \\
367.2 \\
60.45\end{array}$ & $\begin{array}{l}0.045 \\
0.032 \\
1.05 \\
0.003 \\
0.598 \\
0.469 \\
6.499 \\
2.188\end{array}$ & $\begin{array}{l}1,80 \\
1,25 \\
1,37 \\
1,44 \\
0.73 \\
1,56 \\
1,77 \\
3.62\end{array}$ & $\begin{array}{c}2,52 \\
2,55 \\
75,85 \\
0,21 \\
82,3 \\
29,71 \\
360,6 \\
66.4\end{array}$ & $\begin{array}{l}0,080 \\
0,026 \\
1,599 \\
0,003 \\
0,801 \\
0,705 \\
9,886 \\
4,604\end{array}$ & $\begin{array}{l}3,23 \\
1,01 \\
2,11 \\
1,40 \\
0,97 \\
2,37 \\
2,74 \\
6,93\end{array}$ \\
\hline \multicolumn{7}{|l|}{ Normale Konzentration } \\
\hline $\begin{array}{l}\text { Leukocyten }\left(10^{9} / 1\right) \\
\text { Erythrocyten }\left(10^{12} / 1\right) \\
\text { Hämoglobin }(\mathrm{g} / \mathrm{l}) \\
\text { Hämatokrit } \\
\text { MCV }(\mathrm{n}) \\
\mathrm{MCH}(\mathrm{pg}) \\
\mathrm{MCHC}(\mathrm{g} / \mathrm{l}) \\
\text { Thrombocyten }\left(10^{9} / 1\right)\end{array}$ & $\begin{array}{c}7.70 \\
4,80 \\
142,9 \\
0,404 \\
84,1 \\
29.78 \\
353.5 \\
199\end{array}$ & $\begin{array}{l}0,091 \\
0.035 \\
1,182 \\
0.003 \\
0,308 \\
0,224 \\
2,819 \\
2,865\end{array}$ & $\begin{array}{l}1.19 \\
0.73 \\
0.83 \\
0.75 \\
0.37 \\
0.75 \\
0.78 \\
1.44\end{array}$ & $\begin{array}{c}7,82 \\
4,86 \\
142,4 \\
0.411 \\
84,6 \\
29,3 \\
-346,5 \\
217,9\end{array}$ & $\begin{array}{l}0,191 \\
0.070 \\
4,135 \\
0,005 \\
0,994 \\
1,20 \\
12,86 \\
18,63\end{array}$ & $\begin{array}{l}2,44 \\
1,44 \\
2,90 \\
1,26 \\
1,18 \\
4,09 \\
3,71 \\
8,55\end{array}$ \\
\hline \multicolumn{7}{|l|}{ Erhöhte Konzentration } \\
\hline $\begin{array}{l}\text { Leukocyten }\left(10^{9} / 1\right) \\
\text { Erythrocyten }\left(10^{12} / 1\right) \\
\text { Hämoglobin }(\mathrm{g} / \mathrm{l}) \\
\text { Hämatokrit } \\
\text { MCV }(\mathrm{n}) \\
\mathrm{MCH}(\mathrm{pg}) \\
\mathrm{MCHC}(\mathrm{g} / \mathrm{l}) \\
\text { Thrombocyten }\left(10^{9} / \mathrm{l}\right)\end{array}$ & $\begin{array}{c}17.3 \\
6,19 \\
185,1 \\
0,535 \\
86,3 \\
29,92 \\
346,9 \\
642,5\end{array}$ & $\begin{array}{l}0.630 \\
0.085 \\
2.751 \\
0.008 \\
0.47 \\
0,34 \\
3,32 \\
14,21\end{array}$ & $\begin{array}{l}3,64 \\
1,37 \\
1,49 \\
1,44 \\
0.555 \\
1,14 \\
0,92 \\
2,21\end{array}$ & $\begin{array}{c}17,78 \\
6,22 \\
185,4 \\
0,54 \\
86,85 \\
29,82 \\
343,2 \\
-\end{array}$ & $\begin{array}{c}0,814 \\
0,100 \\
6,426 \\
0,009 \\
0,875 \\
1,000 \\
11,56 \\
-\end{array}$ & $\begin{array}{l}4,56 \\
1,61 \\
3,47 \\
1,58 \\
1,01 \\
3,35 \\
3,37 \\
-\end{array}$ \\
\hline
\end{tabular}

der Kontrollproben bedingt. Die Variationskoeffizienten für die verschiedenen Konzentrationsbereiche sind nicht sehr unterschiedlich. Dies bedeutet, daß die Standardabweichung mit steigender Konzentration zunimmt. Die in dieser Arbeit unter Routinebedingungen ermittelten Variationskoeffizienten sind teilweise größer als die von anderen Autoren (1) mit dem gleichen Gerät bei 10 fach-Bestimmung ermittelten Werte. Bei einigen Meß̈größen (Erythrocyten und Hämatokrit) wurde für die Kontrollprobe mit niedriger Konzentration die Streuung in der Serie und von Tag zu Tag gleich groß bestimmt. Eine Erklärung für dieses, von den anderen Kenngrößen abweichende Verhalten, könnten die sehr niedrigen Werte der absoluten Streuung dieser Meßgrößen bei allen drei Kontrollproben sein. Des weiteren ist zu bemerken, daß sich die Mittelwerte für die Thrombocyten bei der Ermittlung der Streuung in der Serie und von Tag zu Tag statistisch signifikant unterscheiden. Dies ist dadurch bedingt, daß für die Ermittlung der Streuung in der Serie und von Tag zu Tag Thrombocytenkontrollproben unterschiedlicher Charge benutzt wurden.

\section{Verschleppung}

Die Möglichkeit einer Verschleppung wurde durch alternierende Messung von Proben hoher und niedriger Konzentration geprüft. Dazu wurde eine Probe mit einer subnormalen Konzentration der Blutkörperchen und des Hämoglobins in 5fach-Bestimmung analysiert. So wurden die Ausgangswerte festgelegt. Anschließend wurde eine Probe hoher Konzentration und die Probe mit subnormaler Konzentration der verschiedenen Kenngrößen 5 mal im Wechsel analysiert. Die Mittelwerte der Meßreihen sind in Tabelle 2 wiedergegeben. 
Tab. 2. Mittelwerte der Fünffachmessung in der Serie (A) einer Probe mit subnormaler Konzentration der zu bestimmenden Kenngrößen sowie die Mittelwerte dieser Probe (B), die fünfmal alterniereñ mit einer Probe höherer Konzentration (C) gemessen wurden.

\begin{tabular}{llllll}
\hline & $\begin{array}{l}\text { Leukocyten } \\
\left(10^{9} / \mathrm{l}\right)\end{array}$ & $\begin{array}{l}\text { Erythrocyten } \\
\left(10^{12} / \mathrm{l}\right)\end{array}$ & $\begin{array}{l}\text { Hämoglobin } \\
(\mathrm{g} / \mathrm{l})\end{array}$ & Hämatokrit & $\begin{array}{c}\text { Thrombocyten } \\
\left(10^{9} / \mathrm{l}\right)\end{array}$ \\
\hline $\mathrm{A}$ & 3,42 & 3,66 & 103,0 & 0,327 & i1 \\
$\mathrm{B}$ & 3,55 & 3,65 & 103,5 & 61,8 \\
$\mathrm{C}$ & 129,70 & 6,29 & 185,0 & 0,326 & 59,1 \\
\hline
\end{tabular}

Wie ein Vergleich der Meßreihen A und B zeigt, sind der Spülvorgang und die Probenverarbeitung im ELT-8 so ausgelegt, daß es nicht zu einer Verschleppung kommt, wobei die Konzentrationsunterschiede der von uns getesteten Proben sich um einen Faktor $2-10$, je nach Kenngröße, unterschieden.
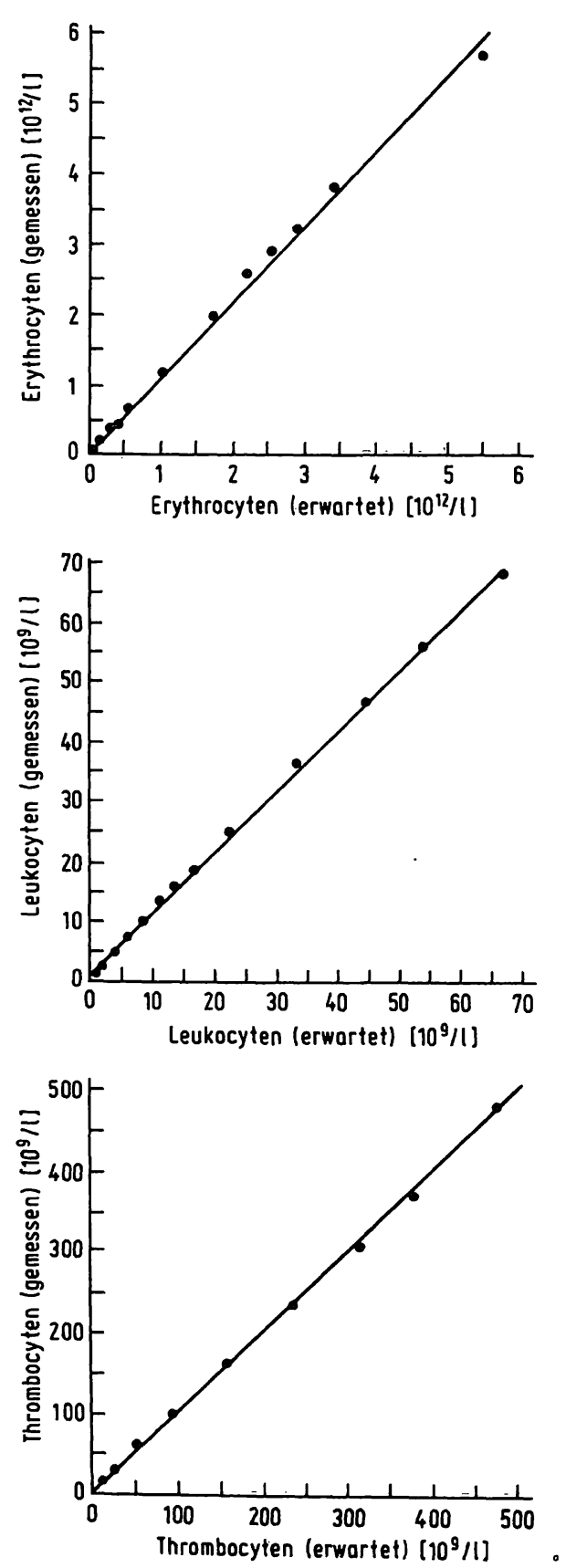

Abb. 1. Die Linearität der verschiedenen Meßgrößen.

\section{Linearität der Methoden}

Für die Leukocyten-, Erythrocyten= und Thrombocytenzählung sowie die Hämoglobin- und Hämatokritbestimmung wurde die Linearität der Messungen über einen möglichst weiten Konżentrationsbereich überprüft. Wie die Daten in Abbildung 1 zeigen,
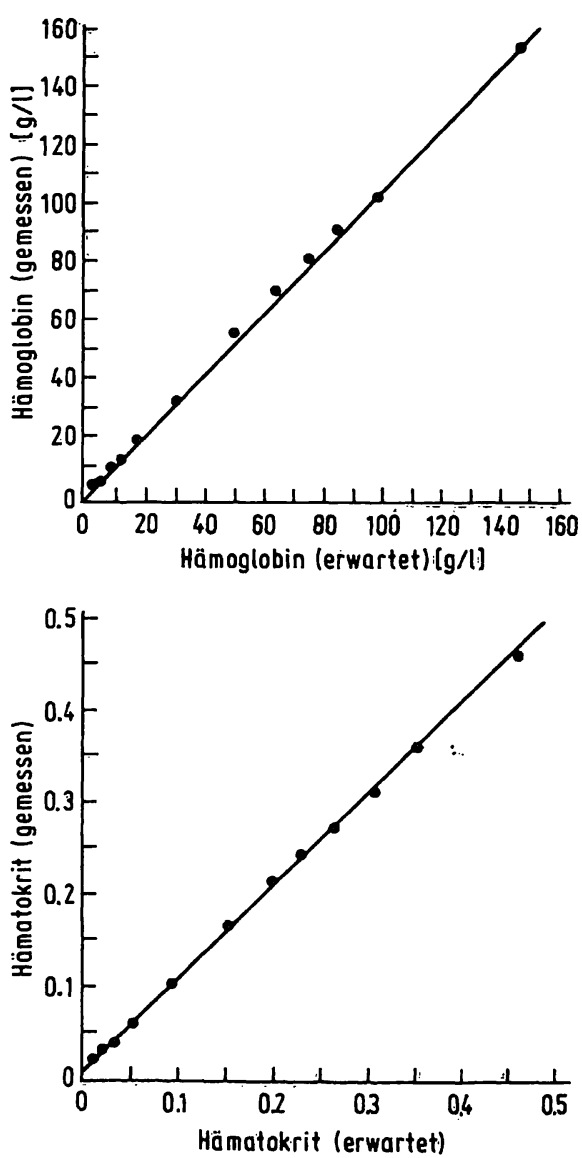
konnte eine Linearität der Messung für die Leukocyten bis zu $70 \times 10^{9} / \mathrm{l}$, die Erythrocyten bis $6 \times 10^{12} / 1$, die Thrombocyten bis $500 \times 10^{9} / 1$, die Hämoglobinkonzentration bis $150 \mathrm{~g} / \mathrm{l}$ und den Hämatokrit bis 0,50 nachgewiesen werden.

Allerdings ist die Linearität nicht unbegrenzt. Die maximalen Meßwerte, ab denen eine Vorverdünnung der Proben bis zum Erhalt eines korrekten Meßwertes erforderlich ist, sind für die Leukocyten $85 \times 10^{9} / 1$, Erythrocyten $8 \times 10^{12} / 1$, die Thrombocyten $10^{12} / 1$ sowie für die Hämoglobinbestimmung $230 \mathrm{~g} / \mathrm{l}$.
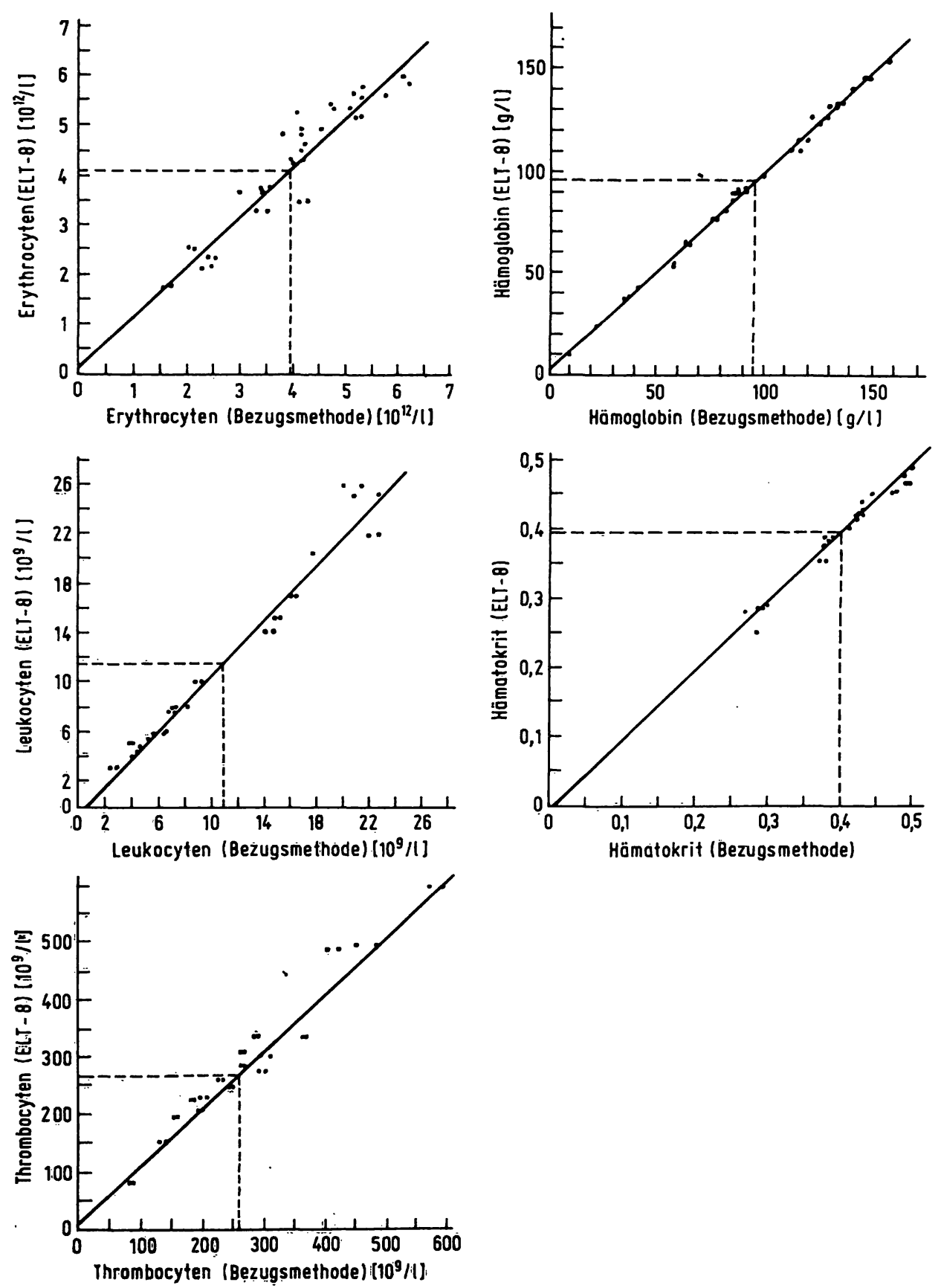

Abb. 2. Vergleichende Bestimmung der Kenngrößen des kleinen Blutbildes mit dem ELT-8 (Ordinate) und Vergleichsverfahren (Abszisse).

J. Clin. Chem. Clin. Biochem. / Vol. 23, 1985 / No. 4

\section{Richtigkeit}

\section{Methodenvergleich}

Die Richtigkeit der Messungen mit dem ELT-8 wurde durch eine Vergleichsmessung der einzelnen KenngröBen von 40 verschiedenen Proben mit konventionellen Verfahren überprüft. Die Hämoglobinbestimmung wurde manuell photometrisch durchgeführt, der Hämotokrit mit der Zentrifuge bestimmt und die Partikelzahlen durch Kammerzählung ermittelt. In Abbildung 2 sind die Meßergebnisse graphisch wiedergegeben. Auf der Ordinate sind die Ergebnisse 
Tab. 3. Statistische Kennzahlen der vergleichenden Messung mit dem ELT-8 Hämatologie-Zellzähler und konventionellen Methoden (Kammerzählung, manuelle photometrische Hämoglobinbestimmung und Hämatokritzentrifuge).

\begin{tabular}{lllll}
\hline Meßgröße & \multicolumn{2}{l}{ Statistische Kenngrößen } & \\
\cline { 2 - 5 } & $\begin{array}{l}\text { Anzahl der } \\
\text { Messungen }\end{array}$ & $\begin{array}{l}\text { Steigung der } \\
\text { Regressionsgerade }\end{array}$ & $\begin{array}{l}\text { Ordinaten- } \\
\text { abschnitt }\end{array}$ & $\begin{array}{l}\text { Korrelations- } \\
\text { koeffizient }\end{array}$ \\
\hline Lcukocyten $\left(10^{9} / 1\right)$ & 40 & 1,116 & $-0,532$ & 0,985 \\
Erythrocyten $\left(10^{12} / 1\right)$ & 40 & 0,992 & 0,194 & 0,978 \\
Thrombocyten $\left(10^{9} / 1\right)$ & 40 & 1,015 & 9,190 & 0,945 \\
Hämoglobin $(\mathrm{g} / \mathrm{l})$ & 40 & 0,967 & 2,758 & 0,987 \\
Hämatokrit & 40 & 0,999 & $-0,008$ & 0,986 \\
\hline
\end{tabular}

mit dem ELT-8 und auf der Abszisse die mit den Vergleichsmethoden aufgetragen. Die statistischen Kenndaten sind in Tabelle 3 zusammengefaßt.

Wie die Korrelationskoeffizienten $(0,945$ bis 0,986$)$ sowie die Steigungen der Regressionsgeraden $(0,967$ bis 1,116$)$ zeigen, ist die Übereinstimmung der ELT8-Messungen 'mit denen der Vergleichsmethoden gut. Eine wichtige Voraussetzung für eine gute Übereinstimmung ist die Kalibrierung des ELT-8 mit einem geeigneten Standard. Die von uns ermittelten Ergebnisse beim Methodenvergleich stimmen mit denen von Raik et al. überein (1).

\section{Einflußgrößen und Störfaktoren}

Neben den Einflüssen der Analytik auf die Meßergebnisse sind Einflüsse, die sich bei der Probennahme, beim Transport und der Verwahrung bemerkbar machen können, zu berücksichtigen. Eine mögliche Einteilung der vielfältigen Einflüsse unterscheidet nach einem Vorschlag von Guder (2) Störfaktoren (in vitro) und Einflußgrößen (in vivo). Eine Zusammenstellung und Diskussion der Literatur der Präanalytik hämatologischer Meßgrößen wurde kürzlich publiziert (3). In den folgenden Ausführungen zu diesem Thema sollen eigene Meßergebnisse und einige Punkte, die wichtig erscheinen, diskutiert werden.

\section{Histogramme}

Der ELT-8-Hämatologie-Zellzähler erlaubt die graphische Darstellung der Größenverteilungen von Leuko-, Erythro- und Thrombocyten. In jüngster Zeit sind einige beschreibende Arbeiten $(11-14)$ zu dieser Fragestellung erschienen. Ein Nachteil der Messungen mit dem ELT- 8 ist, daß bei erhöhten Leukocytenzahlen (ab 15-20 $\left.\times 10^{9} / 1\right)$ die Proben verdünnt werden müssen. Der Maßstab der Ordinate kann nämlich nicht geändert werden, so daß bei erhöhten Leukocytenzahlen das Peakmaximum außerhalb des Ordinatenmaßstabes liegt. Hilfreich kann die Feststellung eines Histogrammes bei Vorliegen von Kälteagglutininen gegen Erythrocyten oder Thrombocyten sein. In diesen Fällen treten im Leukocytenhistogramm im linksseitigen Teil zusätzliche Peaks auf. Thalassämie und Eisenmangelanämie führen zu einer Linksverschiebung und eine megaloblastäre Anämie zu einer Rechtsverschiebung im Histogramm der Erythrocyten.

\section{Einflußgrößen}

Bei der Probennahme ist zu beachten, daß es beim Übergang von einer aufrechten in eine liegende Körperhaltung durch die damit verbundenen Wasserverschiebungen zwischen Intravasalraum und Interstitium zu einer Konzentrationsänderung der makromolekularen und korpuskulären Bestandteile des Blutes kommt. Dies wird auch durch das in folgender Abbildung wiedergegebene Verhalten von Leuko- und Erythrocyten-Konzentration sowie des Hämoglobins wiedergegeben.

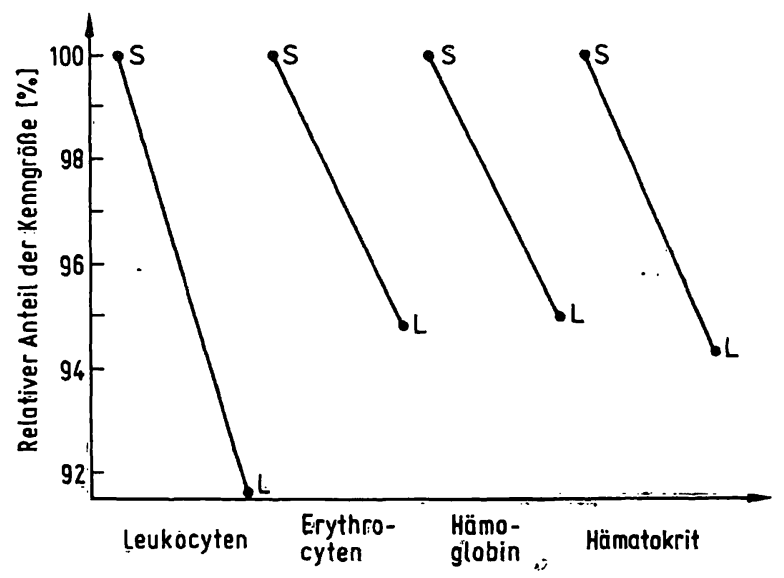

Abb. 3. Prozentuale Abnahme der Leuko- und Erythrocytenzahlen sowie der Hämoglobinkonzentration und des Hämotokrits $10 \mathrm{~min}$ nach dem Übergang vön einer sitzenden (S) in eine liegende Position (L). Aufgetragen sind die Mittelwerte von 18 Proben (4). 
Die von Röcker et al. (4) gefundenen Abweichungen sind durchschnittlich größer als die relativen Standardabweichungen der analytischen Streuung. Konzentrationsänderungen von makromolekularen Bestandteilen des Blutes als Folge einer Änderung der Körperlage liegen in der gleichen Größenordnung von 5-10\% wie von Röcker et al. (4) für die zellulären Bestandteile gefundenen Änderungen (15).

Verschiedene Ursachen können zur einer systematisch zu niedrigen Messung der Thrombocyten führen, zu der sogenannten Pseudothrombopenie. Ohne erkennbare Ursache werden Thrombocytenzahlen kleiner als $50 \times 10^{9} / 1$ bestimmt, während die Funktion der Thrombocyten normal ist. Als Ursachen kommen das Auftreten von Riesenthrombocyten, Kälteagglutininen gegen Thrombocyten und Adhäsionen an neutrophilen Leukocyten in Frage (5-7). Letzteres Phänomen, dessen Ursache unbekannt ist, tritt nur in EDTA-Blut auf. Ebenso kann die Messung der Erythrocyten durch Kälteagglutinine erheblich gestört werden. Deutliche Diskrepanzen zwischen Erythrocytenzahl und Hämoglobinkonzentration, bei Kontrollen stark schwankende Erythrocytenzahlen sowie MCV-Werte ${ }^{3}$ ) größer als 140 fl sollten an Kälteagglutinine denken lassen. Im Differentialblutbild sind entsprechende Agglutinate - ebenso auch bei den Kälteagglutininen gegen Thrombocyten - zu sehen. Häufig kann die Agglutinatbildung durch Erwärmen der Blutprobe auf $37^{\circ} \mathrm{C}$ aufgehoben werden. Abbildung 4 zeigt ein solches Thrombocytenagglutinat.

3) $\mathrm{MCV}=$ mittleres korpuskuläres Volumen.

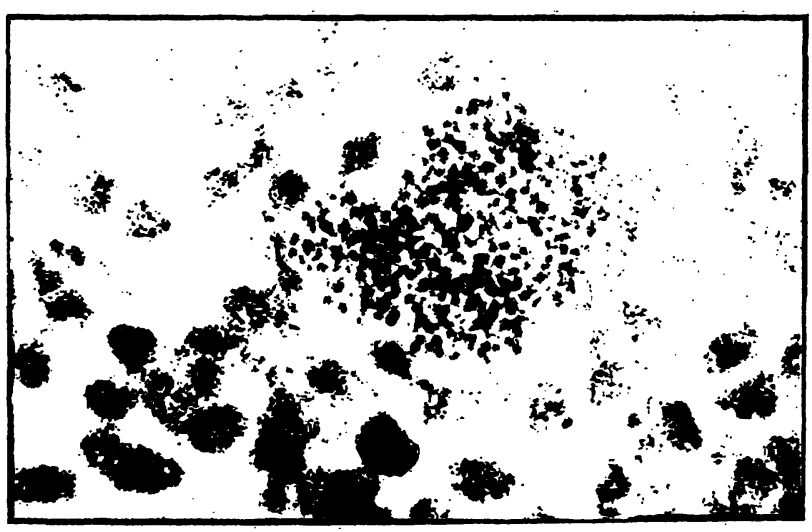

Abb. 4. Thrombocytenagglutinat bei einem Patienten mit Thrombocyten-Kälteautoantikörpern.
Das Prinzip der Zählung der Partikel und ihre Unterscheidung nach Größe bedingt, daß z. B. Agglutinatbildung der Erythrocyten oder Thrombocyten zu einer Erhöhung der Leukocytenmeßwerte führen. Es gibt auch sogenannte Leukoagglutinine, die z. B. nach Antibiotikagabe beobachtet wurden (8), und die zu einer echten Leukopenie führen. Nach unseren Literaturkenntnissen ist aber ein der Pseudothrombopenie ähnlicher Befund für Leukocyten bisher nicht beschrieben. Allerdings wird über Antikörper der Spezifität I und i berichtet (Kälteagglutinine), die eine cytotoxische Wirkung haben und zwischen 7 und $76 \%$ der polymorphkernigen Leukocyten in vitro abtöten (9).

\section{Störfaktoren}

Lipämie oder starke IgM-Erhöhungen führen zu einer Trübung der Reaktionslösung, so daß die Hämoglobinkonzentration systematisch zu hoch bestimt wird. $\mathrm{MCH}$ und $\mathrm{MCHC}^{4}$ ), in deren Berechnung die Hämoglobinkonzentration eingeht, werden ebenfalls zu hoch bestimmt. Nicht gestört werden die Zählung der Erythro-, Leuko- und Thrombocyten $(16,17)$. Die von verschiedenen Untersuchern vorgeschlagenen Methoden zur Beseitigung der Lipämie-Störung bestehen in Zentrifugation (18) oder einer Kombination von Waschen der Zellen mit isotonischer $\mathrm{NaCl}$ Lösung, Zentrifugation und Resuspension der Zellen in NaCl-Lösung vor der Messung (19). Das von Moore et al. (16) für den ELT-8 vorgeschlagene Verfahren sieht eine Messung einer unbehandelten und einer mit Isolac $\left.{ }^{\circledR 5}\right)$ gewaschenen Probe $(500 \mu \mathrm{l})$ vor. Verdünnungsfehler der letzteren Probe werden über einen Quotienten der Erythrocytenzahl der ungewaschenen und gewaschenen Probe korrigiert. Zum Waschen werden $500 \mu \mathrm{l}$ Blut in ein Röhrchen gegeben, der Flüssigkeitsmeniskus mit einem Stift markiert, dann das Röhrchen annährend mit Isolac ${ }^{\circledR}$ gefüllt und die Probe vorsichtig gemischt. AnschlieBend wird die Probe bei $2000 \mathrm{~min}^{-1}$ etwa 5 Minuten zentrifugiert, der Überstand bis zur Strichmarkierung abgezogen und anschließend die Probe gemessen. Der Hb-Wert wird mit Hilfe des Quotienten Erythrocytenzahl (unbehandelte Probe) zu Erythrocytenzahl (gewaschene Probe) multipliziert, um Verluste beim Waschen der Probe auszugleichen. Die verschiedenen Patientenproben wurden nach diesem Vorschlag von Moore et al. (16) untersucht. Die Ergebnisse sind in Tabelle 4 zusammengefaßt.

4) $\mathrm{MCH}=$ mittleres korpuskuläre Hämoglobin. $\mathrm{MCHC}=$ mittlere korpuskuläre Hämoglobinkonzentration.

s) Isolacth, Molter GmbH, Heidelberg. 
Tab. 4. Korrektur des Störeinflusses lipämischer Proben. Die in den ersten drei Abschnitten in Klammern angegebenen Zahlenwerte sind dic Ergebnisse der Kammerzählung.

\begin{tabular}{|c|c|c|c|c|c|c|c|c|c|}
\hline \multirow[t]{2}{*}{ Meßgröße } & \multicolumn{3}{|c|}{ Unbehandelte Proben } & \multicolumn{3}{|c|}{ Gewaschene Proben } & \multicolumn{3}{|c|}{ Endergebnisse } \\
\hline & 1 & 2 & 3 & 1 & 2 & 3 & 1 & 2 & 3 \\
\hline Leukocyten $\left(10^{9} / 1\right)$ & $\begin{array}{c}8.9 \\
(8,6)\end{array}$ & $\begin{array}{c}5,1 \\
(4,9)\end{array}$ & $\begin{array}{c}5,9 \\
(6,1)\end{array}$ & 7,2 & 5,3 & 5,5 & $8,9^{\cdot 1}$ & 5,1 & 5,9 \\
\hline Erythrocyten $\left(10^{12} / 1\right)$ & $\begin{array}{c}4,82 \\
(4,67)\end{array}$ & $\begin{array}{c}5,38 \\
(5,08)\end{array}$ & $\begin{array}{c}4,15 \\
(3,98)\end{array}$ & 4,87 & 4,95 & 4,05 & 4,82 & 5,38 & 4,15 \\
\hline Hämoglobin $(g / l)$ & 153 & 163 & 140 & 152 & 154 & 133 & $\begin{array}{l}151 \\
0456\end{array}$ & $\begin{array}{l}168 \\
0.468\end{array}$ & 135 \\
\hline $\begin{array}{l}\text { Hämotokrit } \\
\text { MCV (fl) }\end{array}$ & $\begin{array}{l}0,456 \\
95\end{array}$ & $\begin{array}{l}0,468 \\
87\end{array}$ & $\begin{array}{l}0,400 \\
96\end{array}$ & $\begin{array}{l}0,445 \\
91\end{array}$ & $\begin{array}{l}0,470 \\
90\end{array}$ & $\begin{array}{l}0,382 \\
94\end{array}$ & $\begin{array}{l}0,456 \\
95\end{array}$ & $\begin{array}{l}0,468 \\
87\end{array}$ & $\begin{array}{l}0,400 \\
96\end{array}$ \\
\hline $\mathrm{MCH}(\mathrm{pg})$ & 31,7 & 30,3 & 33,7 & 31,2 & 31,1 & 32,8 & 31,3 & 31,2 & 32,5 \\
\hline $\mathrm{MCHC}(\mathrm{g} / \mathrm{l})$ & 336 & 348 & 350 & 342 & 328 & 348 & 331 & 359 & $\begin{array}{r}\cdot 338 \\
\end{array}$ \\
\hline Thrombocyten $\left(10^{9} / 1\right.$ & $\begin{array}{c}290 \\
(263)\end{array}$ & $\begin{array}{c}110 \\
(115)\end{array}$ & $\begin{array}{c}214 \\
(208)\end{array}$ & 135 & 62 & 116 & 290 & 110 & 214 \\
\hline
\end{tabular}

Die Triglyceridkonzentrationen lagen zwischen 11 und $26 \mathrm{mmol} / \mathrm{l}$.'Wie die Tabelle 4 zeigt, ist das Verfahren von Moore et al. (16) geeignet, den Störeinfluß durch Lipämie zu korrigieren. Bei ausgeprägterer Lipämie kann es erforderlich sein, den Waschvorgang zu wiederholen.

Eine Hämolyse der Probe - entweder durch unsachgemäße Blutentnahme oder als Folge einer Erkrankung - kann an einer Diskrepanz der Erythrocytenzahl bzw. des Hämatokrits einerseits und der Hämoglobinkonzentration andererseits erkannt werden, da diese in der Regel nicht absedimentiert ins Labor kommen. Heparin kann zu Störungen führen, allerdings erst bei Konzentrationen, die deutlich über den therapeutisch üblichen Konzentrationen von 0,5-2,0 $\mathrm{IU} / \mathrm{ml}$ liegen. Es kommt nämlich bei Zusatz des Hämolysereagenzes zu heparinhaltigen Proben zu Ausfällungen, die zu einer Verstopfung des Gerätes führen.

\section{Probenverwahrung}

Untersuchungen zur Haltbarkeit von Blutproben für die Bestimmung der Parameter des kleinen Blutbildes liegen nur wenige vor. Eine ausführliche Diskussion der bisher vorliegenden Literatur findet sich bei $K$. Schneider (3). Unsere eigenen Untersuchungsergebnisse dazu - mit dem ELT-8 ermittelt - sind in Abbildung 5 wiedergegeben. Aufgetragen sind die Mittelwerte der prozentualen Änderungen gegenüber dem Ausgangswert von 72 verschiedenen Blutproben sowie die entsprechenden Standardabweichungen. Die Proben wurden bei $4^{\circ} \mathrm{C} 4$ Tage lang aufbewahrt. Wie unsere Meßergebnisse zeigen, sind die KenngröBen des roten Blutbildes sehr stabil. Die errechneten
Standardabweichungen für diese Meßgrößen sind ebenfalls sehr klein, d. h. das Verhalten der verschiedenen Blutproben ist einheitlich. Dies stimmt mit den Ergebnissen anderer Untersucher überein $(20,21)$. Dies gilt nicht für die Leukocyten und Thrombocyten. Bei diesen Kenngrößen kommt es im statistischen Mittel zu einem Anfall der gemessenen Anzahl pro Volumeneinheit. Auffällig sind auch die großen Standardabweichungen. Diese zeigen, daß die Änderungen in den verschiedenen Proben, die während der Probenverwahrung auftreten, sehr unterschiedlich sind. Daraus folgt, daß im Einzelfall die Leukocytenund Thrombocytenzahl im Gegensatz zu dem durchschnittlichen Abfall konstant bleiben kann. Dies ist auch eine Erklärungsmöglichkeit, warum ein Untersucher (20) für die Leuko- und Thrombocyten zu

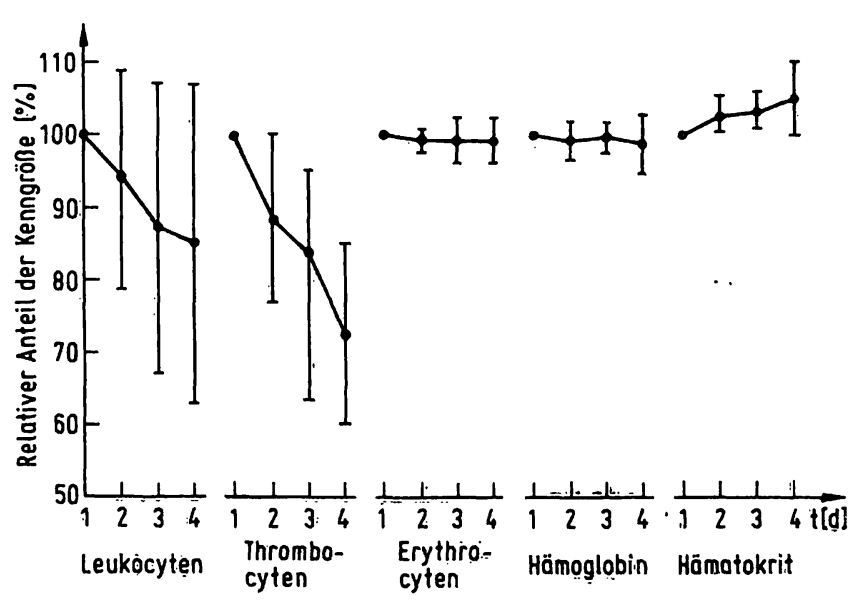

Abb. 5. Mittelwerte und Standardabweichungen der prozentualen Änderung vom Ausgangswert der EDTA-Blutproben - die 4 Tage bei $4^{\circ} \mathrm{C}$ aufbewahrt wurden von 72 verschiedenen Probanden. 
denselben Ergebnissen kommt wie wir, während $\mathrm{Ha}$ milton et al. (21) bei Leukocyten eine Stabilität des Mittelwertes ( $n=104$ Patienten) über 72 Stunden fanden. Allerdings weisen die Autoren darauf hin, $\mathrm{da} B$ die Streuung der Leukocytenmeßwerte mit zunehmender Lagerungsdauer, wie bei unseren eigenen Werten, ansteigen. $\mathrm{Zu}$ berücksichtigen ist, daß je nach Gefäßmaterial sowie der Blutmenge in den Entnahmeröhrchen durch Adsorption Verluste, insbesondere an Thrombocyten, bis zu 15\% erfolgen können (10).

\section{Literatur}

1. Raik, E, McPherson, J., Barton, L., Hewitt, B.S., Powell, E. G. \& Gordon, S. (1980) Pathology 14, 153-164.

2. Guder, W. (1984) Internist 21, 533-542.

3. Schneider, W. (1984) Lab. Med. 7, 136-142.

4. Röcker, L., Schmidt, H.M., Junge, B. \& Hoffmeister, H. (1975) Med. Lab. 28, 267-275.

5. Watkins, jr. St. P. \& Shulman, N.R. (1970) Blood 36, $153-158$

6. Kjeldsberg, C. R. \& Hershgold, E. J. (1974) J. Amer. Med. Ass. 227, 628-630.

7. Greipp, Ph.R. \& Gralnick, H.R. (1976) Blood, 47, $513-521$.

8. Rouveix, B., Lassoued, K., Vittecoq, D. \& Regnier, B. (1983) Brit. Med. J. 287, 1832-1834.

9. Pruzanski, W., Farid, N., Keystone, E. \& Armstrong, M. (1977) Clin. Immunol. Immunopathol. 4, 277-285.

10. Thom, R. (1979) In: Rationalisierung des Medizinischen Laboratoriums (Haeckel, R., ed.), 2. Auflage, GIT-Verlag Ernst Giebeler Darmstadt, 222-295.

11. Scholda, G., Konvacs, Julia, Lanșchützer, Henrike, Unger, W. \& Bayer, P. M. (1983) Lab. Med. 7, 333-335.

\section{Praktikablität}

Die Bedienung des Gerätes einschließlich der Kalibrierung ist einfach. Zur Durchführung kleinerer Wartungsarbeiten ist eine Schulung notwendig. Die Ausfallzeiten betrugen im letzten Jahr etwa 8 Stunden im Monat: Allerdings sind Zeiten für neue Kalibrierung des Gerätes nicht miteinbegriffen. Das Gerät ist so gebaut, daß kleinere Wartungen ohne Sicherheitsrisiko für das Laborpersonal durchgeführt werden können.

12. Lanschützer, Henrike, Konvacs, Julia, Scholda, G., Unger, W. \& Bayer, P. M. (1983) Lab. Med. 7, 336-338.

13. Reheis, Gertraud \& Bayer, P.M. (1983) Lab. Med. 7, $339-341$.

14. von Blohn, G., Jäger, H., Brill, G., Wenzel, E. \& Hellstern, P. (1984) Lab. Med. 8, 5-9.

15. Young, D.S. (1980) In: Chemical diagnosis of disease (Brown, St. \& Mitchell, F. L. \& Young, D.S., eds.), 1st edition, 2nd printing Elsevier/North Holland, Biomedical Press, Amsterdam, New York, Oxford, 56-60.

16. Moore, R., Mahnkovski, V., Pinkey, D.S. \& Lipsey, A. (1984) Lab. Med. 15, 476-477.

17. Lessels, S.E., How, J., Allardyce, M. \& Davidson, R. J. (1978) Med. Lab. Sci. 35, 389-392.

18. Rick, W. (1977) Klinische Chemie und Mikroskopie, 5. Auflage, Springer-Verlag Berlin, Heidelberg, New York, 49.

19. Nicholls, P. D. (1978) J. Clin. Pathol. 30, 638-640.

20. Berg, B., Estborn, B. \& Tryding, N. (1981) Scand. J. Clin. Lab. Invest. $41,425-430$.

21. Hamilton, P.J. \& Davidson, R. L. (1973) J. Clin. Pathol. $26,700-705$.

Prof. Dr. Dr. H. Wisser Abteilung für Klinische Chemie Robert-Bosch-Krankenhaus Auerbachstraße 110 D-7000 Stuttgart 50 
